\title{
Uso De Los Sensores Remotos En Mediciones Forestales
}

\author{
Norma Lara Vásconez. \\ Hernán Chamorro Sevilla \\ ESPOCH / Escuela Superior Politécnica de Chimborazo, \\ Facultad de Recursos Naturales
}

Doi: 10.19044/esj.2018.v14n15p58 URL:http://dx.doi.org/10.19044/esj.2018.v14n15p58

\begin{abstract}
To understand the use of remote sensors in forestry measurements, some of the most relevant definitions have been taken from a wide variety that currently exist, therefore, we will say that these generally play a predominant role in the Dasometry and that all The forest resource requires qualitative and quantitative information regarding the state of the forest and its evolution over time, with sampling that follows one of the existing methods. Historically, modern discipline arises with the invention of flight and the first photographs are obtained from a balloon in the years 1858 and 1859, in 1909 and on board the airplane the first photographic observation is acquired. The first aerial camera was developed in 1915 by J.T.C. Moore, starting the interesting way forward in the techniques of aerial photography using modified combat aircraft giving way to what was called systematic aerial photography in the late 50s. The development on a global scale of the first artificial satellites, allowed remote sensing in the middle of the 20th century on board the first satellite placed in orbit is that of the TYROS series in 1960 by NASA, becoming the pioneer in missions of meteorological observation, which also led to the appearance of satellite image processing, using mathematical procedures such as the Fourier transform. During the 70s missions were promoted with the objective of exploring the solar system and the moon; giving rise to the first spatial photographs taken by Alan B. Shepard rudimentary, Subsequently, Apollo-9 was used for the first multi-spectral experiment in which 4 Hasselblad cameras with different filters were installed. On July 23, 1972, the Landsat project appeared with the launch of the first satellite of the ERTS series (Earth Resources Technology Satellite). Google Earth in the 21st century, introduces online web services making remote sensing accessible to all audiences, with many techniques and processes that allow an image of the earth's surface to be obtained remotely captured by remote sensors located on satellites or airplanes that gather the spectral and
\end{abstract}


spatial relations of objects. Interferometric radar synthetic aperture They are used to producing accurate digital models of large areas of land. LiDAR(An acronym for the English Light Detection and Ranging) is a monochrome active sensor, its mode of operation consists of measuring the distance between the sensor and the target. It is less expensive compared to manual inventory is multi-purpose, allows a complete survey of the study area, is more efficient than photogrammetry. The multi-spectral acquisition is based on the collection and analysis of areas or objects that emit or reflect radiation at a higher level than nearby objects. The quality of the information collected remotely, once the correction of errors through georeferencing with the help of specialized programs, will depend on their resolutions: spatial, spectral, radiometric and temporal.

Keywords: Forest resources; Dasometry; sampling; remote sensing; artificial satellites; electromagnetic spectrum; LiDAR

\section{Resumen}

Para entender el uso de los sensores remotos en las mediciones forestales, se han tomado algunas de las más relevantes definiciones de entre una gran variedad que existen en realidad, por lo tanto, diremos que estos en general desempeñan un papel preponderante en la dasometría y que todo recurso forestal requiere de información cualitativa y cuantitativa del estado del mismo y de su evolución en el tiempo, con muestreos que sigan algunos de los métodos existentes. Históricamente, la disciplina moderna surge con la invención del vuelo y se obtuvieron las primeras fotografías desde un globo en los años 1858 y 1859, en 1909 y a bordo de un avión se adquiere la primera observación fotográfica. La primera cámara aérea fue desarrollada en 1915 por J.T.C. Moore, comenzando el interesante camino de avance en las técnicas de fotografía aérea utilizando aviones de combate modificados dando paso a lo que se llamó la fotografía aérea sistemática a finales de los años 50.El desarrollo a gran escala de los primeros satélites artificiales, permitió que, a mediados del siglo XX, se utilice la teledetección remota a bordo del primer satélite puesto en órbita es el de la serie TYROS en 1960 por parte de la NASA convirtiéndose en el pionero en misiones de observación meteorológica, con lo cual también dio lugar a la aparición del procesamiento de imágenes satelitales, mediante procedimientos matemáticos como la transformada de Fourier. Durante los años 70 se impulsaron misiones con el objetivo de la exploración del Sistema solar y la luna; dando lugar a las primeras fotografías espaciales tomadas por Alan B. Shepard rudimentariamente, posteriormente fue utilizado el Apolo-9 en el primer experimento multiespectral en la que se instalaron 4 cámaras Hasselblad con distintos filtros. 
El 23 de julio de 1972 aparece el proyecto Landsat con el lanzamiento del primer satélite de la serie ERTS (Earth Resources Technology Satellite). Google Earth en el siglo XXI, introduce los servicios web en línea haciendo posible que la teledetección remota esté al alcance todo público, con muchas técnicas y procesos que permiten obtener una imagen de la superficie terrestre de forma remota captada por sensores remotos situados en satélites o aviones que recogen las relaciones espectrales y espaciales de objetos. Los radares interferométricos de apertura sintética, se usan para producir modelos digitales precisos de grandes áreas de terreno. LiDAR (un acrónimo del inglés Light Detection and Ranging) es un sensor activo y monocromático, su modo de operación consiste en la medición de la distancia que existe entre el sensor y el objetivo. Es menos costoso con respecto al inventario manual, es multipropósito, permite un levantamiento completo del área de estudio, es más eficiente que la fotogrametría. La adquisición multiespectral se basa en la recolección y el análisis de áreas u objetos que emiten o reflejan radiación a un nivel superior al de los objetos próximos. La calidad de la información recogida a distancia, una vez realizada la corrección de los errores por medio de la georreferenciación con la ayuda de un programa especializado, va a depender de sus resoluciones: espacial, espectral, radiométrica y temporal.

Palabras-clave: Los recursos forestales; Dasometría; muestreo; teledetección; satélites artificiales; espectro electromagnético; LiDAR

\section{Introducción}

El avance tecnológico en la actualidad y sobre todo en las ciencias informáticas, ha permitido importantes adelantos en las ciencias forestales y más específicamente las mediciones utilizando sensores remotos. De hecho, los sensores remotos en general desempeñan un papel preponderante en las mediciones forestales, permitiendo al profesional forestal clasificar los bosques según su tipo, así como el cálculo de áreas y la elaboración de mapas para facilitar la planificación y ejecución de los planes para el manejo boscoso.

En investigaciones publicadas, establecen que "los recursos forestales requieren de información cualitativa y cuantitativa del estado del mismos y de su evolución en el tiempo. En general, la mensura forestal genera información mayoritariamente cuantitativa, aunque a menudo mezclada con atributos cualitativos". De acuerdo con la FAO (Organización Mundial para la Agricultura y la Alimentación) "Evaluación es el proceso de contextualización de los datos del inventario y de asignación de valores al recurso" (Klein, 2000).

En este documento, se examinará la medición y evaluación forestal que se concentra en árboles y bosques, tanto en medición, cálculo del crecimiento y cambios en los árboles y bosques, con la ayuda de la teledetección. 


\section{Metodología}

La investigación está basada en la literatura de publicaciones anteriores que ofrece una gran variedad de definiciones del uso de los sensores remotos en las mediciones forestales, y cómo estos ayudan a los intelectuales a obtener datos con precisión, claridad y brevedad. Entre los principales conceptos se listan los siguientes:

La medición forestal o dasometría implica la determinación del volumen de árboles completos y de sus partes, las existencias de maderas en rodales, la edad y el incremento de árboles individuales y de rodales completos, así como la magnitud y volumen de sus productos (Romahn, et al., 1994).

Dasometría, en inglés Forest mensuration o bien Forest meansurement, y en el sentido más amplio considera la medida de los bosques. Es una parte de la Dasonomía que se encarga de la medición, cálculo o estimación de los volúmenes, edad e incremento de las masas forestales (Santillán, 1990).

La evaluación forestal también incluye la medición y cálculo del crecimiento y cambio en árboles y bosques utilizando los criterios cualitativos, cuantitativos, directo e indirecto, y es importante por tres razones:

1. Los recursos forestales a pesar de ser un recurso natural renovable tienen un ritmo de crecimiento que puede ser superado por la tasa de aprovechamiento de los mismos.

2. La cuantificación de los recursos forestales permite la toma de decisiones en cuanto a la optimización del uso de suelo, incluida en los planes de manejo forestal.

3. El conocimiento de los recursos forestales permite definir planes de desarrollo regional integrales que incluyen el crecimiento en el sector industrial forestal y de infraestructura productiva y apoyo a las comunidades rurales (caminos forestales y caminos rurales).

La muestra en los inventarios forestales generalmente es una superficie de área, la cual debe cumplir con dos condiciones: que la muestra sea representativa de la población, significa elegir un sitio donde toda la variabilidad de la población se encuentre representada. Que las muestras sirvan para hacer inferencias correctas acerca de la población, lo cual se logra si se seleccionan mediante métodos o procedimientos estadísticos. (eds. Lorena Orozco, Cecilia Brumér, 2002).

En los inventarios forestales, el uso del muestreo mediante el uso de parcelas de área fija es el más utilizado, ya que la probabilidad de selección de los árboles, está relacionada directamente al área de la parcela y a la frecuencia de los individuos que están dentro de ella (Péllico Netto y Brena, 1997).

Existen varios métodos de muestreo forestal así tienen el de área fija, línea o Stand, Prodan, cuadrantes y Bitterlich. Por otro lado, la FAO distingue 
dos tipos de muestreo al azar: simple y estratificado, teniendo como uno de los elementos principales del muestreo la relación porcentual de la superficie de la muestra a la superficie total del área en estudio, como muestra la siguiente relación:

Donde:

$$
f=\left(\frac{n}{N}\right) * 100
$$

$f=$ Intensidad de muestreo en porcentaje

$n=$ Número de unidades de la muestra

$N=$ Número de unidades de toda la población.

En los inventarios forestales se utiliza la intensidad de $0.1 \%$ a $1 \%$ en función de la superficie a inventariar, recursos financieros, precisión requerida y tiempo disponible. Por lo que se puede deducir que en bosques de clima templado-frío es posible utilizar parcelas de muestreo de forma circular, con un radio de $17.84 \mathrm{~m}$. (Figura 1)

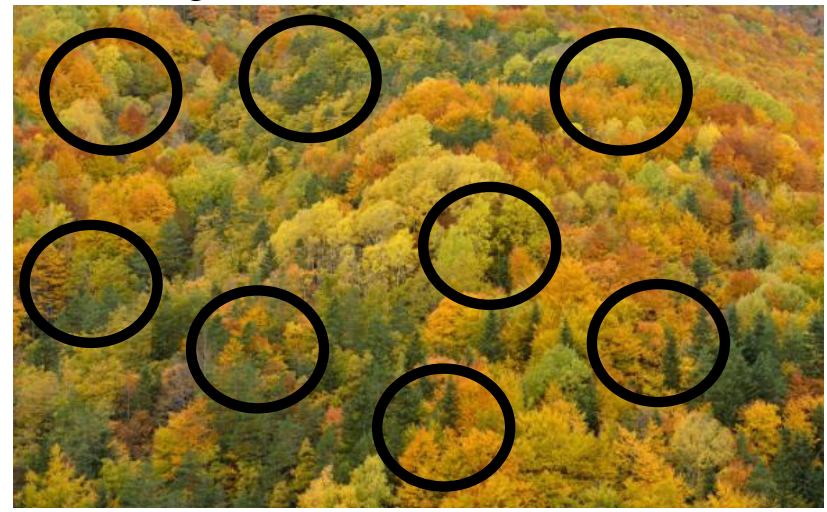

Figura 1. Forma circular de las unidades de muestreo

Por otro lado, en los bosques de clima cálido-húmedo las unidades de muestreo se delimitan con forma rectangular, generalmente con dimensiones de $20 \times 50 \mathrm{~m}$, como se muestra en la Figura 2.

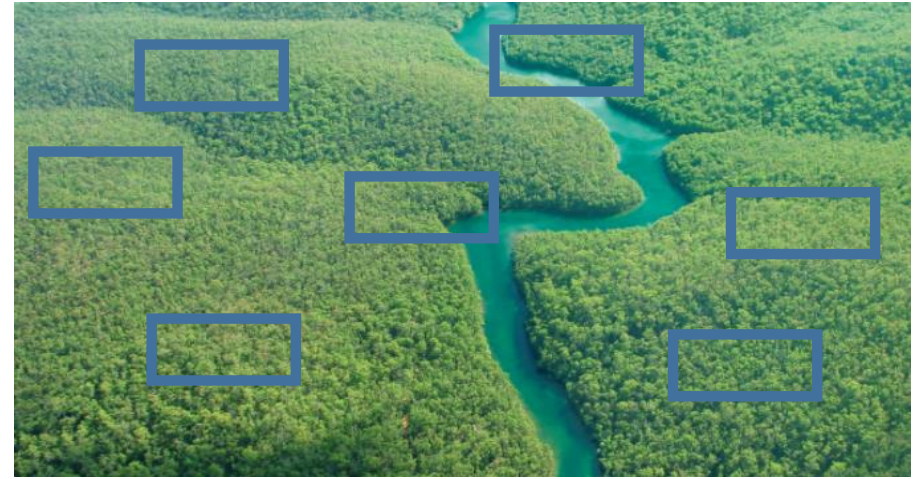

Figura 2. Forma rectangular de las unidades de muestreo. 
La teledetección comienza con los primeros métodos que usaron antiguamente, era subirse a un risco o un árbol para ver el paisaje, posteriormente la disciplina moderna surge con la invención del vuelo. G. Tournachon (alias Nadar), un piloto conocido de globos, tomó fotografías de París desde su globo en 1858. Posteriormente Gaspar Félix obtuvo sus primeras fotografías aéreas desde un globo en 1859, posteriormente se presenta un desarrollo trascendental en la observación fotográfica desde un avión, es así que en el año 1909 Wilbur Wight adquiere la primera fotografía aérea.

La primera cámara aérea fue desarrollada en 1915 por J.T.C. Moore, comenzando el interesante camino de avance en las técnicas de fotografía aérea. El desarrollo de cámaras de reconocimiento, así como también el aparecimiento de nuevos sensores como el radar y los sistemas de comunicación surgen en la segunda guerra mundial. Estas innovaciones encuentran su principal aplicación en la explotación y control de recursos naturales.

A finales de los años 50, se desarrollaron importantes investigaciones como son las fotografías aéreas sistemáticas realizadas desde aviones como el P-51, el P-38, el RB-66 y el F-4C, o algunas plataformas de recolección de información como por ejemplo el U2/TR-1, el SR-71, el A-5 y el OV-1.

El desarrollo de satélites artificiales a mediados del siglo XX permitió el uso de la teledetección remota. El primer satélite puesto en órbita es el de la serie TYROS, en 1960 por parte de la NASA y que es el pionero en misiones de observación meteorológica, con lo cual también dio lugar a la aparición del procesamiento de imágenes satelitales, mediante procedimientos matemáticos como la transformada de Fourier.

En los años 70 se impulsaron misiones a la exploración del sol y la luna; dando lugar a las primeras fotografías espaciales tomadas por Alan B. Shepard de una manera rudimentaria. El Apolo-9 fue utilizado en el primer experimento multiespectral, en el que se instalaron 4 cámaras Hasselblad con distintos filtros, todas estas experiencias permitieron que el 23 de julio de 1972 se realice el lanzamiento del primer satélite de la serie ERTS (Earth Resources Technology Satellite) proyecto conocido como Landsat. 


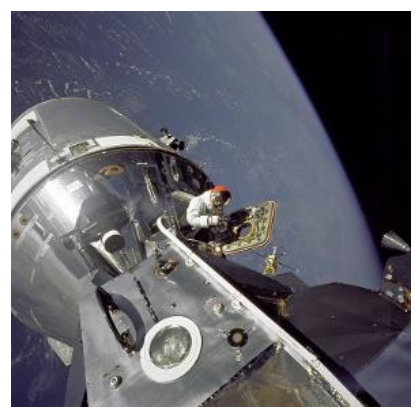

Figura 3. Apollo IX. Fuente:

https://upload.wikimedia.org/wikipedia/commons/thumb/6/62/Gumdrop_Meets_Spider_-

_GPN-2000-001100.jpg/800px-Gumdrop_Meets_Spider_-_GPN-2000-001100.jpg

Posteriormente se lanzaron los satélites SPOT francés, MOS1 y el MODIS del sistema EOS de la NASA, Nimbus y algunos más recientes como el RADARSAT y el UARS, abastecieron de medidas globales de información de varios tipos. Las sondas espaciales ${ }^{2}$ enviadas a otros planetas para la exploración de su entorno, utilizan la teledetección remota; es así que el radar de apertura sintética a bordo del Magellan proporcionó mapas topográficos detallados de Venus, mientras que los instrumentos a bordo del SOHO permitieron estudios del Sol y los vientos solares.

Los satélites de teledetección más comunes a la actualidad son: Satélites de recursos naturales (Landsat y SPOT), Meteorológicos (GOES, NOAA, Seawifs y MODIS), Satélites de alta resolución (Ikonos, Quickbird, Geoeve, WorldView), Equipos radar (ERS, Envisat y Radarsat).

Sensores Remotos o Teledetección es la ciencia y el arte de obtener información de un objeto, área o fenómeno, a través del análisis de datos adquiridos mediante un dispositivo, el cual no está en contacto directo con el objeto, área ni fenómeno que se está investigando (Lillesand y Kiefer, 1994).

Enmarca muchas técnicas y procesos que permiten obtener una imagen de la superficie terrestre de forma remota captada por sensores situados en satélites o aviones que captan las relaciones espectrales y espaciales de objetos, para posteriormente esta imagen ser tratada e interpretada con el objetivo de obtener información de la superficie terrestre y de sus cambios teniendo en cuenta que esta información es global, objetiva, periódica y digital. Un sistema de teledetección espacial se compone de una fuente de energía, cubierta terrestre, sistema sensor (sensor-plataforma), sistema de percepción-comercialización, intérprete y usuario final. (Chuvieco 2008).

Los sensores remotos pueden ser usados en diversas aplicaciones como la agricultura, medioambiente, urbanismo, geología, oceanografía, minería, petróleo, gas, vulcanología, forestación y deforestación utilizando cámara para

\footnotetext{
${ }^{2}$ Una sonda espacial es un dispositivo artificial que se envía al espacio con el fin de estudiar
} cuerpos de nuestro Sistema solar, tales como planetas, satélites, asteroides o cometas 
tomar las fotografías aéreas e imágenes de satélite que operan en uno o más rangos del espectro electromagnético (EEM) emitiendo o reflejando varios tipos de energía como la radiación electromagnética, gravedad, magnetismo, geofísico y onda de radio; así como en longitudes de onda larga que abarcan áreas de mayor superficie para poder detectar una señal.

La fuente de energía, es uno de los elementos importantes dentro del proceso de la obtención de la información, estos sensores adquieren datos a partir de la emisión y reflexión de la "Radiación Electromagnética" (REM), debido a los atributos de la superficie terrestre que puede ser natural (el Sol) o artificial (generada por el propio sensor).

Los sensores activos, como el radar, generan radiación directa hacia un objeto registrando de esa forma los datos, operan en los espectros de microonda y ondas de radio dentro del EEM. Ver Figura 4.

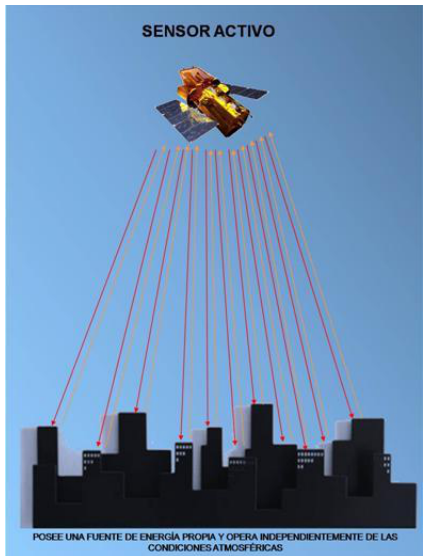

Figura 4. Imagen de un sensor activo. Fuente:

http://www.inegi.org.mx/est/contenidos/proyectos/Preview.aspx

Los sensores pasivos, por su parte registran la radiación del sol, dentro del EEM captando el visible, el infrarrojo cercano, infrarrojo medio, y las longitudes de onda del infrarrojo termal. Ver Figura 5.

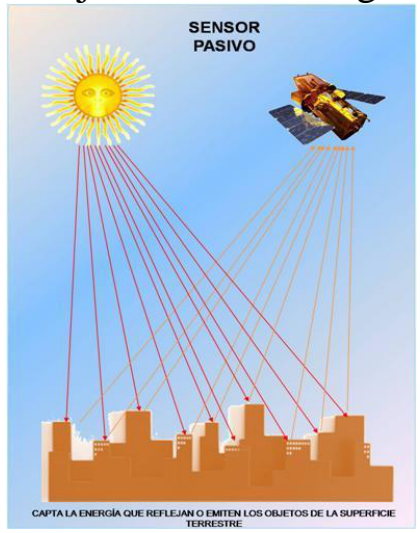

Figura 5. Imagen de un sensor pasivo. Fuente:

http://www.inegi.org.mx/est/contenidos/proyectos/Preview.aspx 
Sin embargo, algunos sensores de microonda son pasivos, así como la fotografía aérea y los satélites Landsat. El registro de datos en estos puede grabarse de 2 formas: fotográfico o digital (numérico), pero para poder ser analizados, deben estar en formato digital para que sean procesados utilizando algún programa especializado, por lo que cualquier dato grabado fotográficamente, necesariamente debe ser convertido a formato digital (numérico).

Los datos obtenidos por los sensores en una amplia variedad de los recursos de la tierra, a partir de la energía electromagnética, son instrumentos que pueden ser colocados en plataformas orbitales o ser aerotransportados, para determinar la resolución espacial y espectral y ser utilizados en la elaboración de mapas, ser cuantificados o monitorizados, incluso determinar la química de los materiales.

Los procesos y elementos que actúan en los sensores remotos electromagnéticos son la adquisición y el análisis de los datos. En la adquisición de los datos involucra: una fuente de energía, propagación de la energía a través de la atmósfera, interacción de la energía con la superficie terrestre, retransmisión de la energía por la atmósfera, sensores orbitales y/o aerotransportados y generación de datos digitales y/o analógicos.

Mientras que el análisis de datos consiste en: el estudio e interpretación tanto analógica como digital, referencia sobre los recursos estudiados (mapa de suelos, afloramientos, revisión de los datos de campo), compilación de toda la información y producto final presentado a los usuarios. Ver Figura 6.

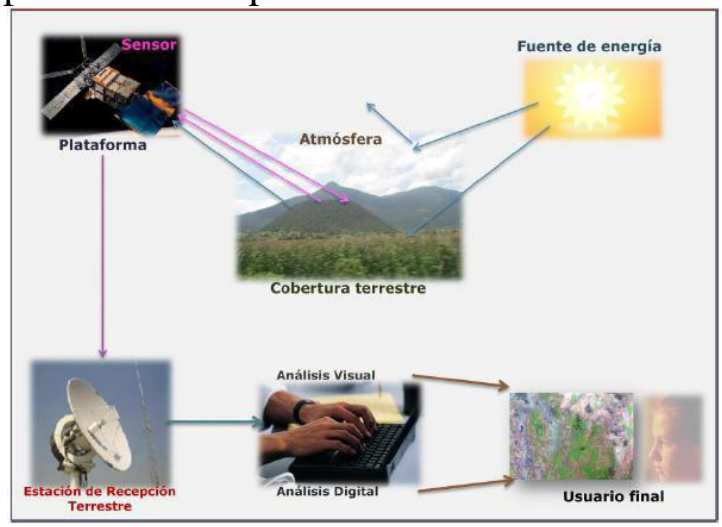

Figura 6. Diagrama de los elementos del proceso de percepción remota o teledetección. Fuente: http://www.inegi.org.mx/est/contenidos/proyectos/Preview.aspx

El radar convencional, se ha asociado principalmente al control del tráfico aéreo, y a la recolección de cierta información meteorológica a gran escala. El radar Doppler es utilizado como apoyo para hacer cumplir con los límites de velocidad locales, así como también de refuerzo a la recolección de información meteorológica como la velocidad del viento y la dirección del 
mismo. El plasma de la ionosfera, es otro tipo de recolector de información activa.

Los radares interferométricos de apertura sintética se usan para producir modelos digitales precisos de grandes áreas de terreno. Los altímetros por láser y radar en los satélites, proveen una gran cantidad de información, midiendo las protuberancias del agua causadas por la gravedad, la velocidad del viento y la dirección, además mapean las características en el fondo del mar en una resolución aproximada de una milla, toman la altura y la longitud de las olas en el océano.

LIDAR (un acrónimo del inglés Light Detection and Ranging) es un sensor activo monocromático y es usado para detectar y medir la concentración de varios elementos químicos en la atmósfera. Ha tenido un desarrollo rápido desde mediados de los 90 . Su modo de operación consiste en la medición de la distancia que existe entre el sensor y el objetivo.

El tiempo que tarda el pulso láser en llegar al objeto con el que impacta y regresar al receptor es transformado en distancia.

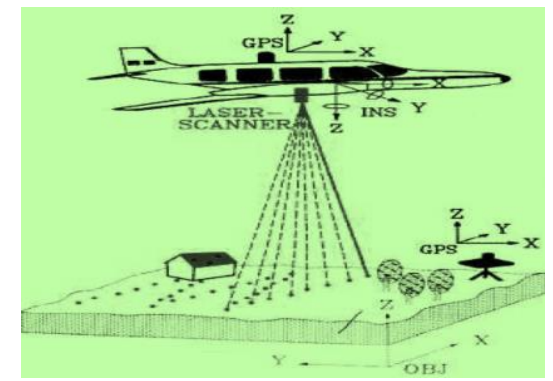

Figura 7. LiDAR sensor activo

[Fuente: http://www.sbgmaps.com/lidar.htm(ACCESO, 18/VI/2009)]

El resultado de un vuelo es una red irregular 3D de puntos $(\mathrm{X}, \mathrm{Y}, \mathrm{Z})$ que describe la forma de la superficie en un momento determinado. El sistema LiDAR está compuesto por un emisor-receptor láser, escáner, GPS diferencial, unidad de medición inercial (IMU), contador de alta precisión y ordenador de a bordo. El comportamiento en zonas forestales, se establece que en superficies sólidas solo tiene un retorno por láser emitido, en el agua la señal del LiDAR topográfico se pierde. Ver figura 8. 


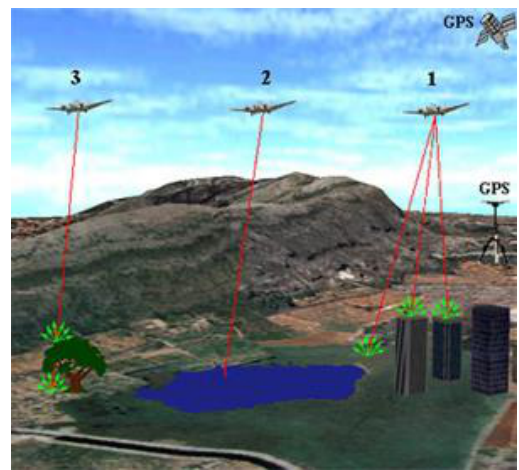

Figura 8. LiDAR en superficies sólidas y agua

[Fuente: http://www.dielmo.com/, (ACCESO, 21/XI/2005)]

Mientras que en las cubiertas forestales son semipermeables y son almacenados dos retornos por pulsado; el primero muchas veces se produce sobre las capas y el segundo algunas veces se produce sobre el suelo. Ver figura 9.

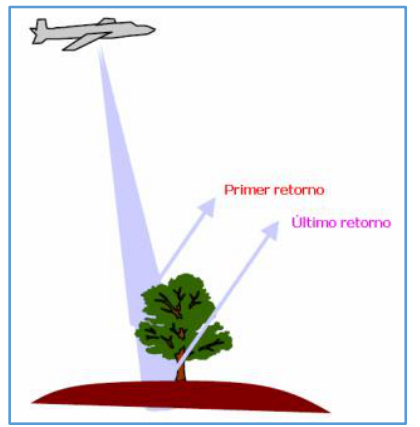

Figura 9. LiDAR en cubiertas forestales

[Fuente: http://www.cfr.msstate.edu/forestry/sitl/research/LiDAR, (ACCESO, 21/IV/2006)]

Las ventajas que LiDAR con respecto al inventario manual es menos costoso, es multipropósito, permite un levantamiento completo del área de estudio frente al muestreo que permiten los trabajos de campo, permite inventarios en zonas inaccesibles y difíciles, es más eficiente que la fotogrametría porque puede describir con eficacia las masas forestales y lo que hay debajo de ellas.

La tecnología LiDAR aporta rapidez en la adquisición de datos, el procesado de datos es automático, facilita la exportación de datos a un SIG, facilita la elaboración de mapas temáticos, posibilidades de elaborar MDEs, MDSs y MDCs de alta resolución, extracción de MDE y del MDAM de calidad, los costos unitarios disminuyen con la superficie escaneada y con el número de objetivos previstos para cada vuelo, en combinación con imagen aérea las posibilidades se multiplican sobre todo en inventario de recursos 
naturales y forestales, ya que las bandas multiespectrales añaden información cualitativa.

Los posibles objetivos Forestales-Ambientales son: Inventario forestal, índices y mapas de vegetación, mapas de pendientes, orientaciones y altitudes de alta resolución, clasificación de tipos de bosques, identificación de especies, cartografía de combustibles, hidrología, captación de $\mathrm{CO}_{2}$, biomasa, clasificación de áreas desforestadas, mapas de vegetación endémica, monitoreo forestal, incendios forestales, detección de cambios y ecología del paisaje.

La calidad de la información recogida a distancia depende de su resolución tanto espacial, espectral, radiométrica y temporal.

Resolución espacial, es el tamaño de un píxel que se guarde en una imagen rasterizada - los píxeles se corresponden con áreas cuadradas cuyo tamaño varía de 1 a 1000 metros. Ver Figura 10.

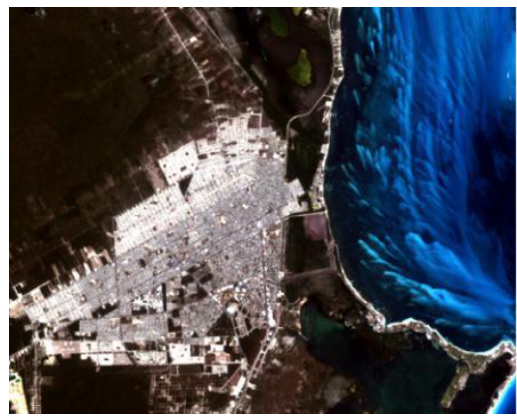

Figura 10. Imagen LANDSAT de la ciudad de Cancún con $30 \mathrm{~m}$ de resolución espacial, formada con las bandas 1,2 y 3 . Fuente:

http://www.inegi.org.mx/geo/contenidos/imgpercepcion/imgsatelite/elementos.aspx

Resolución espectral, es la amplitud de la longitud de onda de las diferentes frecuencias grabadas - normalmente, se relaciona con el número de frecuencias que graba la plataforma. Ver Figura 11.

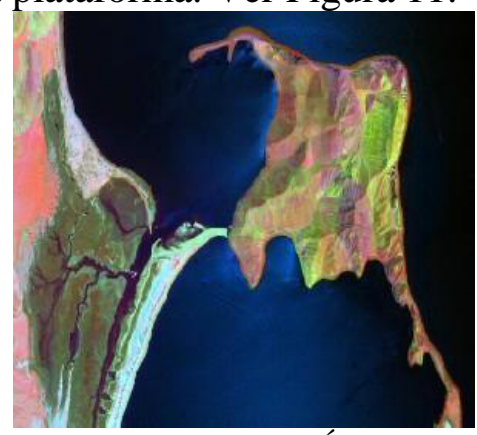

Figura 11. Imagen hiperespectral del sensor MÁSTER que muestra la Isla San Luis

Gonzaga. Fuente: Laboratorio de Cartografía. CICESE. Fuente:

http://www.inegi.org.mx/geo/contenidos/imgpercepcion/imgsatelite/elementos.aspx 
Resolución radiométrica, es la capacidad del sensor para distinguir diferentes intensidades de radiación. Normalmente, comprende de 8 a 14 bits, correspondiente a los 256 niveles de una escala de grises, y puede llegar a 16384 intensidades de color en cada banda. También depende del ruido del aparato. Ver Figura 12.

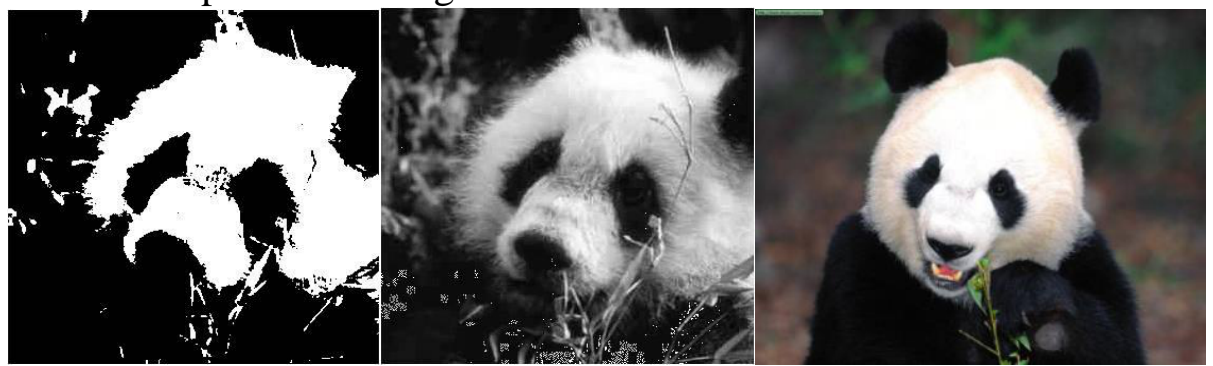

Figura 12. Una sensibilidad de 1BIT, 1 BYTE, 2 BYTES

Resolución temporal, es la frecuencia con la que el avión o satélite sobrevuelan una zona, y solo tiene importancia en estudios para investigar el efecto del paso el tiempo, como en la monitorización de las deforestaciones. El paso de una nube sobre el área u objeto haría necesario volver a repetir el proceso sobre esa zona.

Para poder crear mapas basados en la información recogida por un sensor, la mayoría de los sistemas de teledetección remota extrapolan la información extraída por el sensor de un punto de referencia, incluyendo distancias entre los puntos conocidos en el terreno y va a depender del tipo de sensor usado. En fotografías normales, las distancias son más precisas en el centro de la imagen, las cuales se distorsionan al alejarse del centro de la misma. Otro factor significativo es el rodillo contra el que se ponen las fotos, hecho que puede causar graves errores en las fotografías cuando las mismas se usan para realizan para medir distancias.

La corrección de estos errores se resuelve utilizando la georreferenciación con la ayuda de un programa especializado para relacionar los puntos en la imagen (30 o más por imagen) que se extrapolan usando un punto de referencia establecido previamente, transformando la imagen para producir una información espacial más precisa. Gran parte de imágenes por satélite vendidas estaban totalmente georreferenciadas desde principios de los años 90. Además de esta corrección, las imágenes pueden necesitar una corrección radiométrica y atmosférica. Ver Figura 13. 


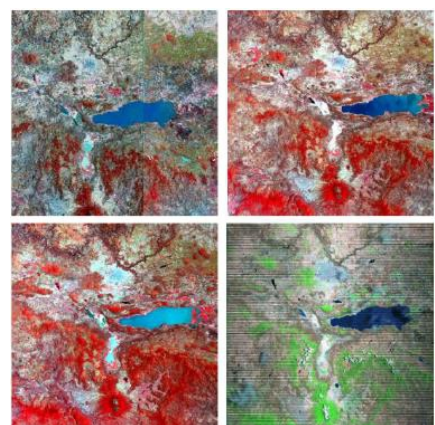

Figura 13. Imágenes LANDSAT de 1976, 1983, 1992 y 2010 que muestran cambios importantes en el nivel del agua en el Lago de Pátzcuaro, Michoacán. Fuente:

http://www.inegi.org.mx/geo/contenidos/imgpercepcion/imgsatelite/elementos.aspx

Una vez procesada la información se procede con la corrección de la misma con las siguientes características:

Corrección radiométrica, da una escala de valores por píxel. Por ejemplo, la escala monocromática de 0 a 255 se convertirá a valores de radiación actuales.

Corrección atmosférica, elimina la neblina atmosférica reescalando cada banda de frecuencia a su valor mínimo (cada píxel a 0). La digitalización de la información también hace posible manipular los datos cambiando valores en la escala de grises. En fotografías aéreas fue aplicada primero la interpretación que es la parte crítica del proceso de hacer la información comprensible. El proceso constaba de:

- Medidas espaciales con el uso de una mesa iluminada, tanto en cobertura convencional simple como en estereográfica.

- Hacer uso de las dimensiones conocidas de los objetos para detectar modificaciones.

- $\quad$ El análisis de imágenes mediante una aplicación automatizada por un programa especializado.

El análisis de objetos basados en imágenes (OBIA - Object-Based Image Analysis) es una subdisciplina de GIScience $^{3}$ dedicada a dividir las imágenes de la teledetección remota en imágenes con significado sobre los objetos, y evaluando sus características en una escala especial, temporal y espectral.

La información antigua obtenida de teledetección remota por lo general es valiosa porque provee de información a largo plazo de una gran porción geográfica. Por otro lado, dicha información es compleja de interpretar y difícil de almacenar; ya que los sistemas actuales almacenan digitalmente sin pérdida de compresión, lo cual hace difícil la interpretación

\footnotetext{
${ }^{3}$ Es la disciplina científica que estudia las estructuras de datos y las técnicas computacionales para capturar, representar, procesar y analizar información geográfica.
} 
de la información por su fragilidad, formato arcaico y propenso a falsificaciones.

Uno de los mejores sistemas para almacenar información es el microfilm ${ }^{4}$, que normalmente sobreviven en librerías comunes, con un periodo de vida de varios siglos. Pueden crearse, copiarse, archivar y almacenar en sistemas automatizados. Son muy compactos como la información almacenada en dispositivos magnéticos y pueden ser leídos con un mínimo de equipo adecuado por el ser humano.

Los niveles de procesamiento de la información, se definieron varios niveles para facilitar dicho procesamiento; es así que en 1986 por la NASA como parte de su Sistema de Observación de la Tierra se adoptaron tanto en la NASA como en el resto de lugares. Los cuales se listan en la tabla 1.

\begin{tabular}{|c|c|}
\hline Nivel & Descripción \\
\hline 0 & $\begin{array}{l}\text { La información científica recogida está a máxima resolución, ordenada } \\
\text { temporalmente y con errores de transmisión, artefactos y duplicados eliminados. }\end{array}$ \\
\hline 1.a & $\begin{array}{l}\text { Información a máxima resolución reconstruida, ordenada cronológicamente y } \\
\text { con anotaciones auxiliares como coeficientes de calibración radiométrica y } \\
\text { geométrica, y parámetros de georreferencia computados y anotados, pero sin } \\
\text { aplicarse al nivel } 0 \text { de información (o, si se aplican, se aplican de tal forma que } \\
\text { ese nivel } 0 \text { se pueda recuperar totalmente del nivel 1.a). }\end{array}$ \\
\hline $1 . \mathrm{b}$ & $\begin{array}{c}\text { La información del nivel 1.a ha sido procesada a unidades de detección; no } \\
\text { todos los instrumentos tienen información del nivel 1.b; la información del nivel } \\
0 \text { es ya irrecuperable. }\end{array}$ \\
\hline 2 & $\begin{array}{l}\text { Variables geofísicas derivadas (altura de las olas del mar, concentraciones de } \\
\text { hielo) a la misma resolución y localización que la información del nivel } 1 .\end{array}$ \\
\hline 3 & Las variables son mapeadas uniformemente en "grids" espacio-temporales. \\
\hline 4 & $\begin{array}{l}\text { Resultado de los análisis de niveles inferiores (variables que no han sido } \\
\text { medidas, pero si han sido derivadas de esas medidas). }\end{array}$ \\
\hline
\end{tabular}

Tabla 1. Niveles de procesamiento de la información

La información guardada en el Nivel 1 es fundamental por ser reversible, ya que tiene un significado científico y una utilidad significativa, y es la base de la generación del resto de niveles. La mayoría de las aplicaciones científicas usan el Nivel 2, debido a que valor es mayor que la del resto de niveles inferiores y es menos pesado que el Nivel 1 debido a que sus parámetros han sido reducidos ni temporal, espacial o espectralmente.

La información almacenada en el Nivel 3 puede ser manipulada sin temor a incurrir en un manejo inadecuado de los datos, ya que es bastante más pequeña, general y útil que el resto de niveles y que es utilizada por la mayoría

${ }^{4}$ Es un sistema de archivo, gestión y difusión documental. Su producción está sometida a un alto grado de normalización, tanto en su metodología como en los materiales utilizados y un estricto control de productos resultantes. 
de las aplicaciones. La organización temporal y espacial de este nivel hace más factible poder combinar la información de otras fuentes. Toda la información es procesada en programas especializados que se listan a continuación:

La información recogida por la teledetección remota es procesada y analizada utilizando el programa especializado de su preferencia, ya que existe un gran número de aplicaciones de código abierto, y otras tantas de pago, para procesar esta clase de información. De acuerdo con el estudio NOAA, realizado por Global Marketing Insights, Inc. Se tienen los porcentajes como muestra la Tabla 2:

\begin{tabular}{|c|c|}
\hline Aplicación & Porcentaje \\
\hline ESRI & $30 \%$ \\
\hline ERDAS IMAGINE & $25 \%$ \\
\hline ITT Visual Information Solutions (ENVI) & $17 \%$ \\
\hline MapInfo & $17 \%$ \\
\hline ERMapper & $11 \%$ \\
\hline total & $100 \%$ \\
\hline
\end{tabular}

Tabla 2. Porcentaje de procesamiento y análisis NOAA.

Fuente: https://es.wikipedia.org/wiki/Teledetecci\%C3\%B3n

Entre las academias occidentales, el estudio constató estos otros porcentajes como muestra la tabla 3:

\begin{tabular}{|c|c|}
\hline Aplicación & Porcentaje \\
\hline ESRI & $39 \%$ \\
\hline ERDAS IMAGINE & $27 \%$ \\
\hline ITT Visual Information Solutions (ENVI) & $17 \%$ \\
\hline MapInfo & $9 \%$ \\
\hline AutoDesk & $7 \%$ \\
\hline Otras & $1 \%$ \\
\hline total & $100 \%$ \\
\hline
\end{tabular}

Tabla 3. Porcentaje de procesamiento y análisis - academias occidentales. Fuente: https://es.wikipedia.org/wiki/Teledetecci\%C3\%B3n

Otros paquetes de aplicaciones en relación con la teledetección remota incluyen PCI Gemoatics que desarrolla PCI Geomática, un paquete de aplicaciones relacionadas con la teledetección remota líder en Canadá, IDRISI de los laboratorios Clark, y el programa eCognition de Definiens. Algunas aplicaciones de código abierto son: GRASS GIS, QGIS, OSSIM, Optics, SPRING y Orfeo toolbox.

\section{Medición y cálculo de área de bosque}

En el manejo forestal, hay dos momentos durante los cuales es importante medir y calcular el área de un bosque. 
El primer momento es antes de empezar la planificación del manejo y el inventario general. Difiere según el tamaño características y formas de los bosques, para así calcular la disponibilidad total de madera por ciclo de corta.

El segundo momento es para medir área de bosque es antes de la planificación del aprovechamiento, cuando ya se ha decidido cuanto y donde se va aprovechar cada año. Hay que medir y marcar el área de corta anual (ACA) como unidad productiva y administrativa En cada unidad de corta se realiza después de un censo comercial para medir todo los arboles comerciales a partir de un diámetro predeterminado.

Permiten al profesional forestal clasificar los bosques según tipos, calcular sus respectivas áreas y elaborar mapas para facilitar la planificación y ejecución de los planes de manejo forestal. Dos ejemplos de sensores remotos son: cámaras para tomar las fotografías aéreas e imágenes de satélite.

Los sensores miden datos acerca de un objeto pueden obtenerse por medio de ondas electromagnéticas que emiten o reflejan. Los sensores remotos usan detectores de energía para registrar variaciones.

La mayoría de los sensores operaran en uno o más rangos del espectro electromagnético, también operan en longitudes de onda larga abarcan área de mayor superficie para poder detectar una señal, por ejemplo, los sensores de calor.

En el manejo forestal ha sido usado para detectar enfermedades en el bosque, los sensores de calor también se usan para detectar áreas con diferentes tipos de vegetación y grados de humedad.

\section{Resultados:}

Como resultado de la investigación se tiene que los recursos forestales requieren de información de cualitativa y cuantitativa, en relación con el estado de los mismos y de su evolución en el tiempo, con el cálculo de los volúmenes, edad e incremento de las masas forestales por medio del muestreo forestal que es el acto de tomar o seleccionar una muestra del bosque, se realizan mediciones sobre la muestra para aplicar los resultados a todo el bosque.

La utilización del método tradicional en inventarios grandes como a nivel nacional y regional incide en el costo de mantener brigadas de trabajo, el tiempo, el personal técnico con cierto grado de capacitación en el uso de diferentes instrumentos de medición.

Cuando se realizan mediciones forestales áreas inaccesibles como bosques húmedos tropicales o en las montañas donde el acceso dificulta realizar un inventario satisfactorio.

En la actualidad las mediciones forestales se pueden obtener con el uso de los sensores remotos permitiendo clasificar los bosques según el tipo, 
calcular áreas y elaborar mapas para facilitar la planificación y ejecución de los planes de manejo boscoso.

La teledetección se basa en muchas técnicas y procesos que permiten obtener una imagen de la superficie terrestre de forma remota captada por sensores situados en satélites o aviones, los mismos operan en uno o más rangos del espectro electromagnético permitiendo captar las relaciones espectrales y espaciales de objetos y materiales a una distancia considerable y son aprovechados en diversas aplicaciones, para generar información detallada con los procesos de adquisición y análisis; y son conocidos como sensores activos y pasivos.

Durante el proceso de adquisición y análisis de áreas y objetos se utiliza técnicas multiespectrales entre las que se tiene el radar convencional, plasma de la ionosfera, altímetros por láser y radar en los satélites, lindar, radiómetros y fotómetros, fotografía estereoscópica, LandSat, geodesia, acústica y semiacústica y sistema de navegación Inercial.

Una vez obtenida la información, se procede con el procesamiento de la misma por medio de la resolución espacial y espectral, Landsat 8, resolución radiométrica y resolución temporal, para posteriormente realizar la corrección de la misma con la ayuda de la "corrección radiométrica o la corrección atmosférica" y finalmente poder ser analizada con la ayuda de programas especializados como ESRI, ERDAS IMAGINE, ENVI, MapInfo, ERMapper, AutoDesk entre otros.

Los datos obtenidos mediante sensores remotos permiten llevar el historial de un bosque gracias a su resolución temporal, permitiendo el manejo forestal en la detección de enfermedades en el bosque, así como la detección de áreas con diferentes tipos de vegetación y grado de humedad.

Para mediciones forestales utilizando sensores tele transportados en bosques húmedos tropicales de difícil acceso resultan favorables. De la misma forma cuando se trabaja en grandes extensiones de bosques resulta beneficioso el utilizar la obtención de la información mediante sensores remotos.

Podemos asumir que los datos de teledetección óptica ganarán importancia en las Evaluaciones Forestales Nacionales futuras

\section{Conclusiones}

La dasometría en la actualidad ha tomado un giro importante ya que sus resultados podrán ser más eficientes y efectivos, gracias a la tecnología actual para mediciones forestales como son los sensores remotos que ayudan en la recolección de la información proveniente de la cubierta forestal. Tal es el caso de LiDAR que es un sensor activo y monocromático que el proceso de muestreo es menos costos que el muestreo manual.

La tecnología LiDAR aporta rapidez en la adquisición de datos y el procesado de los mismos es automático facilitando la exportación a un SIG, 
dentro del cual se puede elaborar mapas temáticos, la elaboración de MDEs, MDSs y MDCs de alta resolución, la extracción de MDE y del MDAM de calidad, reduciendo costos a gran escala, estos datos se pueden combinar con imágenes aéreas sobre todo en inventario de recursos naturales y forestales ya que las bandas multiespectrales añaden información cualitativa.

Por todo lo antes expuesto concluiremos que el uso de los sensores remotos en las mediciones forestales, y el papel que desempeñan dentro del proceso de obtención de la información; cualitativa y cuantitativa para que sea analizada utilizando usando algún programa SIG, ayudando de esta manera a los investigadores forestales a tomar la mejor decisión.

\section{References:}

1. Green Forest Evaluación de los Recursos Forestales. (1998). http://www.virtual.chapingo.mx. http://www.virtual.chapingo.mx/dona/sis.prod.forestal/Evaluaci\%F3n .pdf

2. Revista, D., Universidad, R., Vega-Araya, M., Rica, C., BonattiGonzález, J., \& Rica, U. D. C. (2018). Vega-Araya, 6 (2016), 1-6. Recuperado a partir de http://www.revistas.una.ac.cr/index.php/dialogo/index

3. Espinoza, G., Geraldine, G., Magaña, G., Jesús, J., Ramos, H., Flores, M., ... Ramos, H. (2016). Precisión de los coeficientes y cocientes de forma en la estimación del volumen de Pinus montezumae Lamb. Accuracy of form coefficients and quotients in estimating the volume of Pinus montezumae Lamb. Recuperado a partir de http://www.redalyc.org/articulo.oa?id=63448564003

4. Vega-Araya, M., \& Bonatti-González, J. (2016). Monitoreo forestal con sensoramiento remoto en el marco del cambio climático. Universidad en Diálogo: Revista de Extensión. https://doi.org/10.15359/udre.6-2.4

5. Mallén, C. (2012). Revista mexicana de ciencias forestales. Journal of Chemical Information and Modeling. https://doi.org/10.1017/CBO9781107415324.004

6. Núñez-Villalba, J. (2017). Monitoreo espacial de desastres con imágenes de satélite. Recuperado a partir de http://www.geografia.umsa.bo/documents/437373746/0/Monitoreo+ Espacial+de+Desastres+con+imágenes+de+satélite+\%28libro\%29

7. Nacional, X. V. C. (2012). Aplicaciones Forestales de la Tecnología LiDAR, 2012. Recuperado a partir de http://www.congresos.cchs.csic.es/TIG2012/sites/default/files/Aplica ciones_forestales.pdf 
8. INEGI. (2011). Instituto Nacional de Estadística y Geografía. Imágenes de Satélite. Recuperado a partir de http://www.inegi.org.mx/geo/contenidos/imgpercepcion/ImgSatelite/ Elementos.aspx

9. Orozco, L., \& Brúmer, C. (2002). Inventarios Forestales Para Bosques Latifoliados En América Central. Inventarios forestales para bosques latifoliados en América Central. Recuperado a partir de http://orton.catie.ac.cr/repdoc/A4575e/A4575e.pdf

10. Koch, B. (s. f.). La teledetección como apoyo a los inventarios forestales nacionales EFN. Recuperado a partir de http://www.fao.org/fileadmin/user_upload/national_forest_assessmen t/images/PDFs/Spanish/KR2_ES_8_.pdf

11. Mcroberts, R. E., Tomppo, E. O., \& Czaplewski, R. I. (1992). Diseños de muestreo de las Evaluaciones Forestales Nacionales. Antología de conocimiento para la evaluación de los Recursos Forestales Nacionales, 1-21.

12. Forestales, I. (s. f.). Forestales, 1-7. Recuperado a partir de https://www.u-

cursos.cl/forestal/2010/1/MF004/1/material_docente/bajar?id_materi $\mathrm{al}=483201$ 